\title{
The international geoscience school syllabus and its development
}

\author{
Keele University, Keele, Staffordshire, ST5 5BG. E-mail: c.j.h.king@keele.ac.uk
}

(Received January 25, 2014: Revised form accepted November 11, 2014)

International organisations focussed on school-level Earth science education decided in 2012 to develop a recommended geoscience syllabus for use in countries across the world. The syllabus was developed on these initial principles: it should be based on the content of existing curricula, be well-structured, be presented as a succinct document with flexible exemplification, and be given as a list of content to be covered by the age of 16. A range of national Earth science curricula submitted by international colleagues was analysed. A first draft of the syllabus was developed from this analysis and circulated for comment; this was modified in response to feedback and recirculated; the final document was endorsed by the organisations and published on their websites in January 2014. This document has the potential to enhance Earth science education across the world, providing crucial background to those who will become the decision-makes of the future.

\section{Background}

An international survey of the school-level geoscience education across 34 countries (King, 2012, 2013) found that the compulsory Earth science content of national curricula was very variable. Following a wide invitation, the 34 countries which responded to the survey were: Argentina, Australia, Bangladesh, Belgium, Brazil, Canada, Czech Republic, England, Estonia, Finland, France, Germany, India, Indonesia, Israel, Italy, Japan, Korea, Malawi, New Zealand, Norway, Philippines, Portugal, Romania, Russia, Saudi Arabia, Scotland, South Africa, Spain, Sri Lanka, Taiwan, Trinidad \& Tobago, Uruguay and the United States.

One country had no compulsory Earth science whilst in the other countries the content varied from minimal to comprehensive. Earth science was taught through science, geography or a combination of science and geography, whilst the level of teaching varied from only being at primary level to systematic coverage from primary to secondary level and beyond.

It was because of the variability of Earth science education discovered by the survey that a decision was made at meetings of the
International Geoscience Education Organisation (IGEO) and the International Union of Geological Science Commission on Geoscience Education (IUGS-COGE) held at the $34^{\text {th }}$ International Geological Congress held in Brisbane, Australia in 2012, to prepare and publish a recommended syllabus for school-level geoscience education.

A previous attempt to highlight the big ideas of Earth science to be taught in school was formulated and published in the United States (Earth Science Literacy Initiative, 2010), but this was not based on existing curricula or appears to be widely known about beyond the United States.

The following account shows how syllabus was developed and published as an internal report by IGEO and IUGS-COGE (King, 2014).

\section{Principles of syllabus development}

The decision was made to base the syllabus on the content of existing Earth science curricula around the world, since such a syllabus was most likely to be widely accepted. Although the structure of the Earth science content of many of the syllabuses around the world was not readily apparent, the new International Syllabus would have clear structure. The syllabus would be presented in a very concise form, since a short syllabus would be most persuasive to future syllabus developers around the world, who were considering the incorporation of Earth science content - so the core syllabus is presented on a single page. However, this brevity may lead to misunderstanding about the meaning of some of the concepts included, so these would be exemplified in a way allowing different countries to develop different emphases to the syllabus. Such an approach would not allow educational progression to be shown - so the syllabus would simply be presented as a series of Earth science content statement for pupils to encounter by the age of 16 .

The core syllabus developed on these principles is shown in Table 1 and the core syllabus with exemplification is given in Table 2.

\section{Methodology}

Members of the International Geoscience Education Organisation (IGEO) and the International Union of Geological Science Commission on Geoscience Education (IUGS-COGE) were invited to submit the syllabuses being taught in their countries, and nine countries did so (Australia, England, Japan, New Zealand, Norway, 
Table 1. The International Geoscience Syllabus, to be encountered by all pupils by the age of 16 - core syllabus

By the age of 16 pupils should develop an understanding of the following:

Earth as a changing system

- Attributes open to energy, almost closed to matter, changing over time, within the solar system, comprising geosphere, hydrosphere, atmosphere, biosphere

- Interactions interaction of geosphere, hydrosphere, atmosphere, biosphere

- Feedback positive and negative

- Processes and water cycle, rock cycle, carbon cycle products

- Energy sources solar, internal

Earth is a system within the solar system, within the universe

- Origins big bang; accretion from dust; stars; planets

- The Sun only external energy source; fluctuations

- Rotational day/night, seasons, moon phases, eclipses effects

Earth is a system which has changed over time

- Geological time span, major events, relative and absolute dating methods, rates of processes

Earth's system comprises interacting spheres - geosphere

- Earth materials minerals, fossils, sedimentary, igneous and and properties

- Earth processes and preserved characteristics

- Structure of the Earth and evidence

- Plate tectonics and evidence

\section{metamorphic rocks, soil}

surface processes, sedimentary, igneous and

metamorphic processes, deformation

crust, mantle, core, lithosphere

unifying theory, plate construction and subduction, characteristics of plate margins, mechanism, rates of movement; evidence
- hydrosphere

- Continental water

- Oceanic water

- atmosphere

- Composition

- Flow

- Change

- biosphere

- Evolution

- Impact on other systems

Earth's system produces resources

- Raw materials naturally concentrated, non-renewable, uses, need and fossil fuels careful managing (sustainable development), potentially polluting issues

- Renewable energy

Human/Earth system interactions

- Natural hazards human impact, forecasting, mitigation

- Environmental local to global, mitigation issues

- Impact on resource wars; migration due to climate change human history

Earth's system is explored through fieldwork and practical work

- Observation

- Synthesis of observations

- Investigation and hypothesistesting observation, measurement and recording interpretation

devising and implementing plans, processing data, drawing conclusions, evaluating results and communicating findings
Portugal, Scotland, South Africa and the United States of America). The existing syllabus for the International Earth Science Olympiad (IESO) was added. Meanwhile national curriculum developments were taking place in England and the United States at the time, and the documents containing recommendations on the Earth science content submitted for consideration in those developments were also included (shown with a grey background in the analysis).

A tabulated analysis of all these curricula was developed, based on the structure of the IESO syllabus. The Earth science coverage of each syllabus was added to the table, indexed by page numbers of the syllabus. A penultimate column was added to the table indicating the frequency of the items found across the syllabuses as: $\mathrm{F}=$ frequent (more than 4$) ; \mathrm{M}=$ moderate $(3$ or 4$)$; blank = infrequent $(2$ or less).

The new draft syllabus was prepared on the basis of this analysis, and cross-checked with the syllabus analysis table by adding a final column, indicating that the new syllabus addressed the items identified in the survey. The results of this analysis are shown in Tables 3 - 9 and the sources of the national syllabus information used are shown in Table 10.

The draft syllabus was circulated to members of IGEO and IUGSCOGE for comment and generated thoughtful and constructive feedback about the structure, scope and detail of the draft syllabus. The syllabus was modified in the light of this feedback and the second draft was similarly circulated, generating further constructive criticism, particularly from those listed in the Acknowledgements section below.

The final draft of the syllabus was endorsed by the Senior Officers of both the IGEO and IUGS-COGE and published on their respective websites in January 2014.

\section{Comment}

At the outset of the endeavour to produce an international geoscience syllabus, the point was made that it was very easy to write such a syllabus, but much more difficult to agree the outcome with colleagues across the world. However, whilst sometimes providing 
Table 2. International Geoscience Syllabus, to be encountered by all pupils by the age of 16 - core syllabus with exemplification

By the age of 16 , pupils should develop an understanding of the following:

Exemplification of the core to indicate the extent of coverag

(it is anticipated that this will vary from country to country)

\section{Earth as a changing system}

- Attributes

- Interactions

- Feedback

positive and negative

- Processes and products

water cycle, rock cycle, carbon cycle

- Energy sources atmosphere, biosphere open to energy, almost closed to matter, changing over time, within the solar system, comprising geosphere, hydrosphere,

interaction of geosphere, hydrosphere, atmosphere, biosphere causes waves and atmospheric warming; atmosphere/biosphere interaction climatically controls vegetation; lithosphere/biosphere interaction affects soil quality; rates vary from fast to slow positive - increasing area of polar ice sheets gives increased reflection of solar energy, gives increased cooling, gives increasing area of polar ice sheets; negative - the more carbon dioxide is released into the atmosphere, the more that is absorbed in the oceans

unique properties of water, evaporation, transpiration, condensation, precipitation; weathering/erosion, sedimentation, metamorphism, melting, igneous activity; photosynthesis, respiration, burial as limestone/fossil fuel, release by burning/weathering

internal energy from radioactivity and energy from Earth's formation

Earth is a system within the solar system, within the universe

- Origins big bang; accretion from dust; stars; planets

only externich

- Rotational effects solar energy driving the water cycle and weather; long term fluctuations of energy from the Sun related to climate change

major events: 4600 million years (Ma) - formation of Earth. 3600Ma - early life. 550Ma - animals with hard parts; 250Ma - major extinction, including trilobites; 65Ma - major extinction, including dinosaurs; 1 Ma ice age; dating principles: superposition, cross-cutting relationships, fossil correlation; radiometric dating; processes occur on a frequency-magnitude spectrum from continuous to catastrophic

\section{Earth's system comprises interacting spheres -}

- geosphere

- Earth materials and

properties

minerals, fossils, sedimentary, igneous and metamorphic rocks, soil

- Earth processes and preserved characteristic

- Structure of the Earth and evidence

- Plate tectonics and evidence surface processes, sedimentary, igneous and metamorphic processes, deformation

crust, mantle, core, lithosphere

unifying theory, plate construction and subduction, characteristics of plate margins, mechanism, rates of movement; evidence definitions of: mineral, fossil, rock sedimentary rock, igneous rock, metamorphic rock, soil; minerals including: quartz, feldspar, mica, garnet, calcite, halite, gypsum, pyrite, galena; fossils including: trilobite, ammonite, dinosaur; fossilisation processes including: burial, replacement, moulds and casts, trace fossils; rock texture, porosity, permeability; sedimentary rocks including: limestone, chalk conglomerate, sandstone, clay, shale, rock salt; sedimentary features including: layering (bedding), cross bedding, ripple marks; igneous rocks including: granite, basalt, andesite, gabbro, volcanic ash; metamorphic rocks including: slate, schist, gneiss, marble, metaquartzite (quartzite)

weathering (physical/chemical), erosion, transportation, deposition, lithification, metamorphism, intrusion, extrusion, folding, faulting, jointing seismic evidence

constructive, destructive and conservative margins; past and present evidence 
Table 2 Contd....

Earth's system comprises interacting spheres - Contd...

- hydrosphere

- Continental water location, processes of movement, uses

- Oceanic water composition, processes of movement

surface water, groundwater, ice caps/glaciers; infiltration, downhill flow; water resource management salinity; surface flow and waves caused by wind; deep flow due to density differences caused by temperature and salinity

- atmosphere

- Composition

evolution, current composition

outgassing by early volcanic activity; nitrogen, oxygen, trace gasses including water vapour and carbon dioxide

processes of movement

greenhouse effect, planetary influences, human influence,

unequal heating of Earth, flow due to density differences caused by temperature, oceanic heat source temperature graphs over different time spans; link between temperature change and sea level

- Change impact on sea level

palaeogeographical effects on evolution; mass-extinction by volcanic activity and impact biological weathering; biological deposition

- biosphere

- Evolution

natural selection, fossil evidence, mass-extinction role of biosphere in Earth systems

oil/gas; metal ores; bulk raw materials; local examples of mining/quarrying

\section{Earth's system produces resources}

- Raw materials an

naturally concentrated, non-renewable, uses, need careful

fossil fuels

managing (sustainable development), potentially polluting

issues

- Renewable energy

low pollution, cost, regularity of supply

Human/Earth's system interactions

- Natural hazards human impact, forecasting, mitigation

- Environmental issues local to global, mitigation

eruption; earthquake; tsunami; landslide

global human impact (causing erosion, pollution, drainage-changes mining/quarrying); burning fossil fuels and greenhouse effect

- Impact on human history resource wars; migration due to climate change

Earth's system is explored through fieldwork and practical work

- Observation

observation, measurement and recording

- Synthesis of observations

interpretation

devising and implementing plans, processing data,

Investigation and

drawing conclusions, evaluating results and

communicating findings 
Table 3. Matrix of coverage by current school-level Earth science syllabuses - geoscience skills and abilitie

Matrix developed from current syllabuses (and recommendations - US and England)

* Note for Tables 3 - 9: Curriculum recommendations shown in grey columns - not yet implemented

** Note for tables 3 - 9: $\mathrm{F}=$ frequent (more than 4); $\mathrm{M}=$ moderate ( 3 or 4 ); blank = infrequent (2 or less)

\begin{tabular}{|c|c|c|c|c|c|c|c|c|c|c|c|c|c|c|}
\hline Statements - derived from the IESO syllabus & IESO & $\begin{array}{l}\text { Austra- } \\
\text { lia N/C }\end{array}$ & $\begin{array}{c}\text { England } \\
\mathrm{N} / \mathrm{C}\end{array}$ & $\begin{array}{l}\text { Japan } \\
\text { N/C }\end{array}$ & $\begin{array}{c}\text { New } \\
\text { Zealand } \\
\text { N/C }\end{array}$ & $\begin{array}{l}\text { Norway } \\
\text { N/C }\end{array}$ & $\begin{array}{l}\text { Scotland } \\
\text { N/C }\end{array}$ & $\begin{array}{c}\text { South } \\
\text { Africa } \\
\text { N/C }\end{array}$ & $\begin{array}{c}\text { US } \\
\text { stan- } \\
\text { dards }\end{array}$ & $\begin{array}{l}\text { Portugal } \\
\text { N/C }\end{array}$ & $\begin{array}{c}* \text { *US } \\
\text { frame } \\
\text { work }\end{array}$ & $\begin{array}{l}* \text { England } \\
\text { rec. }\end{array}$ & $\begin{array}{c}* * \\
\text { Fre } \\
\text { quency }\end{array}$ & $\begin{array}{c}\text { Covered } \\
\text { by } \\
\text { proposal }\end{array}$ \\
\hline 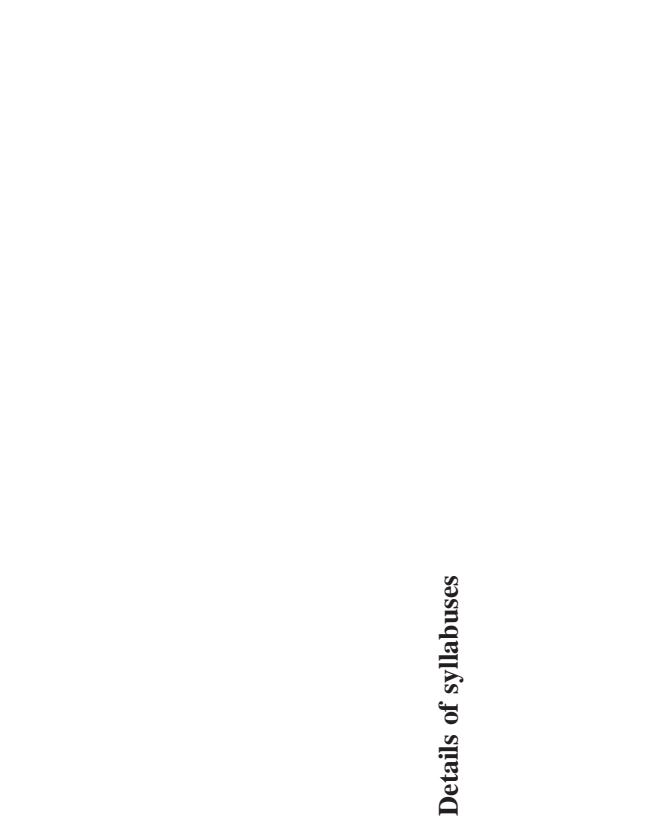 & 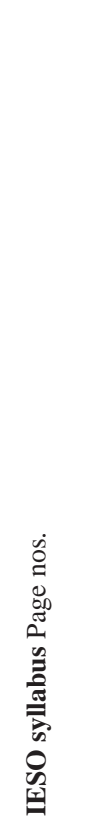 & 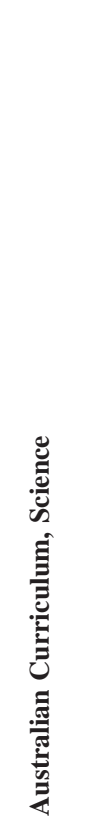 & 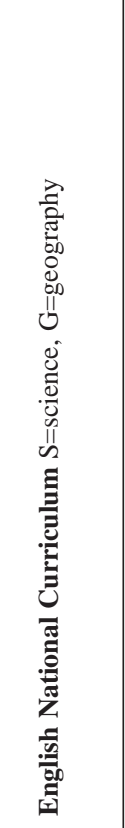 & 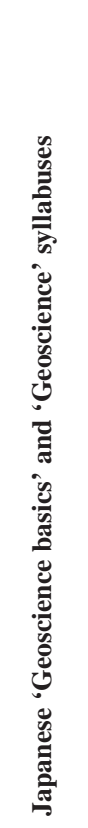 & 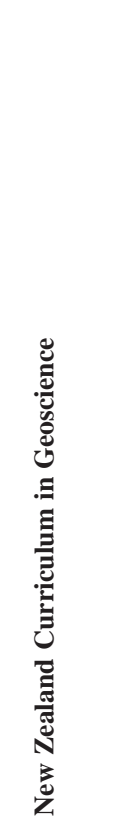 & 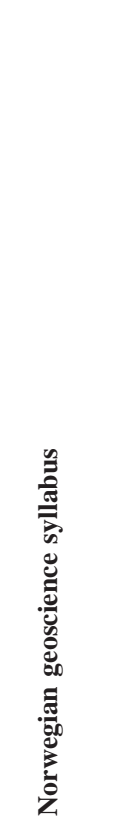 & 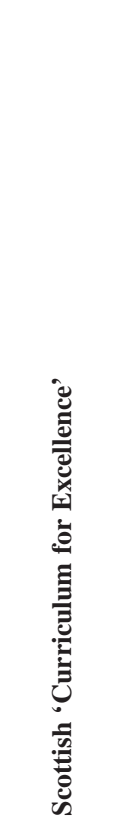 & 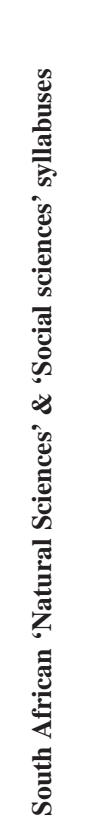 & 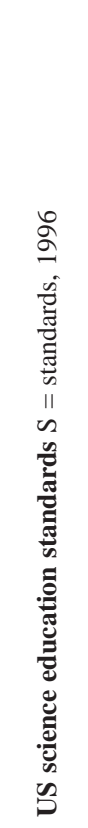 & 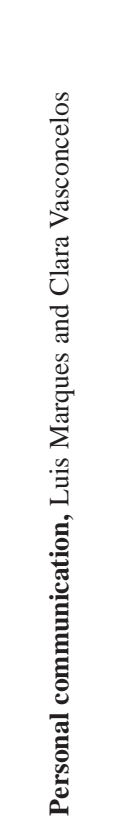 & 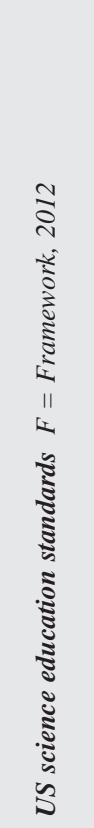 & 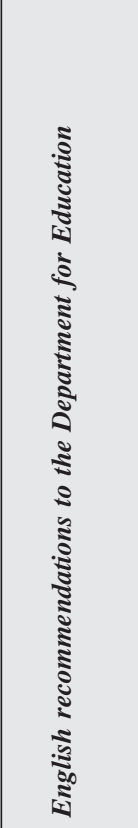 & 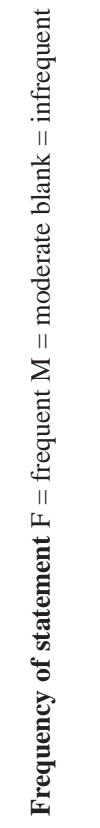 & 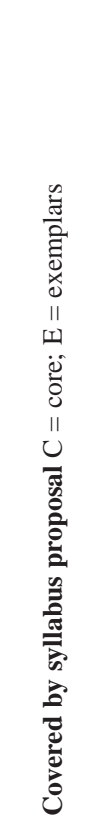 \\
\hline \multicolumn{15}{|l|}{ Geoscience skills and abilities } \\
\hline three dimensional thinking & 4 & & & & & & & & & $\mathrm{x}$ & & & & \\
\hline $\begin{array}{l}\text { thinking on different timescales } \\
\text { including deep time }\end{array}$ & 4 & & & & & & & & & $\mathrm{X}$ & $F 2, F 3$ & & M & $\mathrm{C}$ \\
\hline $\begin{array}{l}\text { thinking at different scales, } \\
\text { from microscopic to global }\end{array}$ & & & & & & & & & & $\mathrm{X}$ & $F 3$ & & & \\
\hline cyclic thinking & 4 & & & & & & & & & $\mathrm{X}$ & & & & \\
\hline systems thinking & 4 & & & & & & & & & $\mathrm{x}$ & $F 3$ & & M & $\mathrm{C}$ \\
\hline field skills & 4 & & $\begin{array}{c}\mathrm{G}, \mathrm{KS} 3 \\
104,107\end{array}$ & & & & & SS 48 & & $X$ & & & M & C \\
\hline $\begin{array}{l}\text { construction of a geological } \\
\text { history }\end{array}$ & 4 & & & & & & & & & $\mathrm{x}$ & $F 2, F 3$ & & M & $\mathrm{C}$ \\
\hline
\end{tabular}


Table 4. Matrix of coverage by current school-level Earth science syllabuses - the geosphere

\begin{tabular}{|c|c|c|c|c|c|c|c|c|c|c|c|c|c|c|c|c|}
\hline \multicolumn{3}{|c|}{ Statements - derived from the IESO syllabus } & IESO & $\begin{array}{l}\text { Austra- } \\
\text { lia N/C }\end{array}$ & $\begin{array}{c}\text { England } \\
\mathrm{N} / \mathrm{C}\end{array}$ & $\begin{array}{c}\text { Japan } \\
\text { N/C }\end{array}$ & $\begin{array}{c}\text { New } \\
\text { Zealand } \\
\text { N/C } \\
\end{array}$ & $\begin{array}{l}\text { Norway } \\
\text { N/C }\end{array}$ & $\begin{array}{c}\text { Scotland } \\
\text { N/C }\end{array}$ & $\begin{array}{c}\text { South } \\
\text { Africa } \\
\text { N/C }\end{array}$ & $\begin{array}{l}\text { US } \\
\text { stan- } \\
\text { dards }\end{array}$ & $\begin{array}{l}\text { Portugal } \\
\text { N/C }\end{array}$ & $\begin{array}{c}* \text { US } \\
\text { frame } \\
\text { work }\end{array}$ & $\begin{array}{l}\text { * England } \\
\text { rec. }\end{array}$ & $\begin{array}{c}* * \\
\text { Fre } \\
\text { quency } \\
\end{array}$ & $\begin{array}{c}\text { Covered } \\
\text { by } \\
\text { proposal }\end{array}$ \\
\hline \multicolumn{3}{|c|}{ The Geosphere } & 6 & 4 & & & & & & & & & & & & \\
\hline $\begin{array}{l}\text { Earth } \\
\text { materials }\end{array}$ & Minerals & & 7 & 3 & & & & S6 & S17 & & & & & & M & $\mathrm{C}$ \\
\hline & & $\begin{array}{l}\text { quartz } \\
\text { orthoclase } \\
\text { plagioclase } \\
\text { biotite } \\
\text { muscovite } \\
\text { garnet } \\
\text { calcite } \\
\text { clay } \\
\text { halite } \\
\text { gypsum } \\
\text { pyrite } \\
\end{array}$ & $\begin{array}{l}7 \\
7 \\
7 \\
7 \\
7 \\
7 \\
7 \\
7 \\
7 \\
7 \\
7 \\
\end{array}$ & & & & & & & & & $\begin{array}{l}X \\
X \\
X \\
X \\
X \\
X\end{array}$ & & & & \\
\hline & Soil & & 7 & & S, KS2 11 & & & & S17 & & & & & $\checkmark$ & M & $\mathrm{C}$ \\
\hline & & $\begin{array}{l}\text { soils have properties of color } \\
\text { and texture, capacity to retain } \\
\text { water, and ability to support } \\
\text { growth of plants, including } \\
\text { those in our food supply }\end{array}$ & & & & & & & & NS 69 & $\mathrm{~S} 1$ & & & & & \\
\hline & & $\begin{array}{l}\text { soil consists of weathered rocks } \\
\text { and decomposed organic } \\
\text { material from dead plants, } \\
\text { animals, and bacteria. Soils are } \\
\text { often found in layers }\end{array}$ & & & & & & & & $\begin{array}{l}\text { NS } 70 \\
\text { NS } 70\end{array}$ & $\begin{array}{l}\mathrm{S} 2 \\
\mathrm{~S} 2\end{array}$ & & & & & $\begin{array}{l}\mathrm{E} \\
\mathrm{E}\end{array}$ \\
\hline & Rock & & 7 & & $\begin{array}{l}\text { S, KS1 } \\
5 \& \text {, } \\
\text { KS2 } 11\end{array}$ & & & S6 & S17 & & & & & & M & $\mathrm{C}$ \\
\hline & & texture & & & S, KS2 11 & & & & & NS 69 & & $\mathrm{X}$ & & & M & $\mathrm{E}$ \\
\hline & & permeability & & & S, KS2 11 & & & & & & & & & & & \\
\hline & Sediment: & y rocks & 7 & 3 & & & & & & NS 70 & & $\mathrm{X}$ & & $\checkmark$ & $\mathrm{F}$ & $\mathrm{C}$ \\
\hline & & $\begin{array}{l}\text { limestone } \\
\text { chalk } \\
\text { chert } \\
\text { clay } \\
\text { marl } \\
\text { dolomite } \\
\text { sandstone } \\
\text { phosphorite } \\
\text { gypsum } \\
\text { rock salt }\end{array}$ & $\begin{array}{l}7 \\
7 \\
7 \\
7 \\
7 \\
7 \\
7 \\
7 \\
7 \\
7 \\
\end{array}$ & & & & & & & & & $\begin{array}{l}\mathrm{X} \\
\mathrm{X}\end{array}$ & & & & \\
\hline
\end{tabular}




\begin{tabular}{|c|c|c|c|c|c|c|c|c|c|c|c|c|c|c|c|}
\hline \multicolumn{2}{|c|}{ Statements - derived from the IESO syllabus } & \multirow{2}{*}{$\begin{array}{c}\text { IESO } \\
7 \\
7\end{array}$} & \multirow{2}{*}{$\begin{array}{c}\text { Austra- } \\
\text { lia N/C } \\
3\end{array}$} & \multirow{2}{*}{$\begin{array}{l}\text { England } \\
\mathrm{N} / \mathrm{C}\end{array}$} & $\begin{array}{c}\text { Japan } \\
\text { N/C }\end{array}$ & $\begin{array}{c}\text { New } \\
\text { Zealand } \\
\text { N/C }\end{array}$ & \multirow{2}{*}{\begin{tabular}{c|} 
Norway \\
N/C
\end{tabular}} & \multirow{2}{*}{$\begin{array}{l}\text { Scotland } \\
\text { N/C }\end{array}$} & \multirow{2}{*}{$\begin{array}{l}\text { South } \\
\text { Africa } \\
\text { N/C } \\
\text { NS } 70\end{array}$} & \multirow{2}{*}{$\begin{array}{c}\text { US } \\
\text { stan- } \\
\text { dards } \\
\end{array}$} & \multirow{2}{*}{$\begin{array}{c}\text { Portugal } \\
\text { N/C } \\
\mathrm{X}\end{array}$} & \multirow{2}{*}{$\begin{array}{l}* \text { *US } \\
\text { frame } \\
\text { work }\end{array}$} & \multirow{2}{*}{$\begin{array}{l}\text { * England } \\
\text { rec. }\end{array}$} & \multirow{2}{*}{$\begin{array}{c}\begin{array}{c}* * \\
\text { Fre } \\
\text { quency }\end{array} \\
\mathrm{F}\end{array}$} & \multirow{2}{*}{$\begin{array}{c}\begin{array}{c}\text { Covered } \\
\text { by } \\
\text { proposal }\end{array} \\
\mathrm{C}\end{array}$} \\
\hline Igneous & & & & & & & & & & & & & & & \\
\hline & $\begin{array}{l}\text { granite } \\
\text { rhyolite } \\
\text { obsidian } \\
\text { basalt } \\
\text { andesite } \\
\text { gabbro } \\
\text { tuff }\end{array}$ & $\begin{array}{l}7 \\
7 \\
7 \\
7 \\
7 \\
7 \\
7 \\
7\end{array}$ & & & & & & & & & $\mathrm{X}$ & & & & \\
\hline Metame & ic rocks & 7 & 3 & & & & & & NS 70 & & $\mathrm{X}$ & & $\checkmark$ & $\mathrm{F}$ & $\mathrm{C}$ \\
\hline & $\begin{array}{l}\text { schist } \\
\text { gneiss } \\
\text { marble } \\
\text { metaquartzite (quartzite) }\end{array}$ & $\begin{array}{l}7 \\
7 \\
7 \\
7\end{array}$ & & & & & & & & & $\begin{array}{l}X \\
X\end{array}$ & & & & \\
\hline Fossils & & 7 & & & & & & & & & & & $\checkmark$ & & \\
\hline & $\begin{array}{l}\text { various forms of fossilisation } \\
\text { fossils provide evidence about } \\
\text { the plants and animals that } \\
\text { lived long ago and the nature } \\
\text { of the environment at that time }\end{array}$ & 7 & & & & & & & $\begin{array}{l}\text { NS 63, } \\
70,72\end{array}$ & S1, S2 & $\begin{array}{l}X \\
X\end{array}$ & & & M & E \\
\hline & mass extinction & & & & & & & & NS 65 & & $\mathrm{X}$ & & & & \\
\hline \multicolumn{16}{|l|}{ Rock structures } \\
\hline Sedimer & y textures/ structures/ features & 7 & & & & & & & & & $\mathrm{X}$ & & & & \\
\hline & $\begin{array}{l}\text { layering (bedding) } \\
\text { graded bedding } \\
\text { cross bedding } \\
\text { ripple marks } \\
\text { discontinuity planes }\end{array}$ & $\begin{array}{l}7 \\
7 \\
7 \\
7 \\
7\end{array}$ & & & 1,2 & & & & & & $\mathrm{X}$ & & & M & $\mathrm{E}$ \\
\hline Igneous & tures/ structures/ features & 7 & & & & & & & & & & & & & \\
\hline & $\begin{array}{l}\text { porphyritic } \\
\text { pegmatitic } \\
\text { scoria } \\
\text { volcano } \\
\text { lava flow } \\
\text { dyke } \\
\text { sill }\end{array}$ & $\begin{array}{l}7 \\
7 \\
7 \\
7 \\
7 \\
7 \\
7\end{array}$ & & & & & & & & & $\begin{array}{l}X \\
X\end{array}$ & & & & \\
\hline Metamo & ic textures/ structures/ features & 7 & & & & & & & & & $\mathrm{X}$ & & & & \\
\hline & $\begin{array}{l}\text { foliation } \\
\text { lineation }\end{array}$ & $\begin{array}{l}7 \\
7 \\
\end{array}$ & & & & & & & & & & & & & \\
\hline Structu & features & 7 & & & & & & & & & & & & & \\
\hline & $\begin{array}{l}\text { fold } \\
\text { fault }\end{array}$ & $\begin{array}{l}7 \\
7\end{array}$ & & & & & & & & & $\begin{array}{l}X \\
X\end{array}$ & & & & \\
\hline
\end{tabular}


Table 4. Contd....

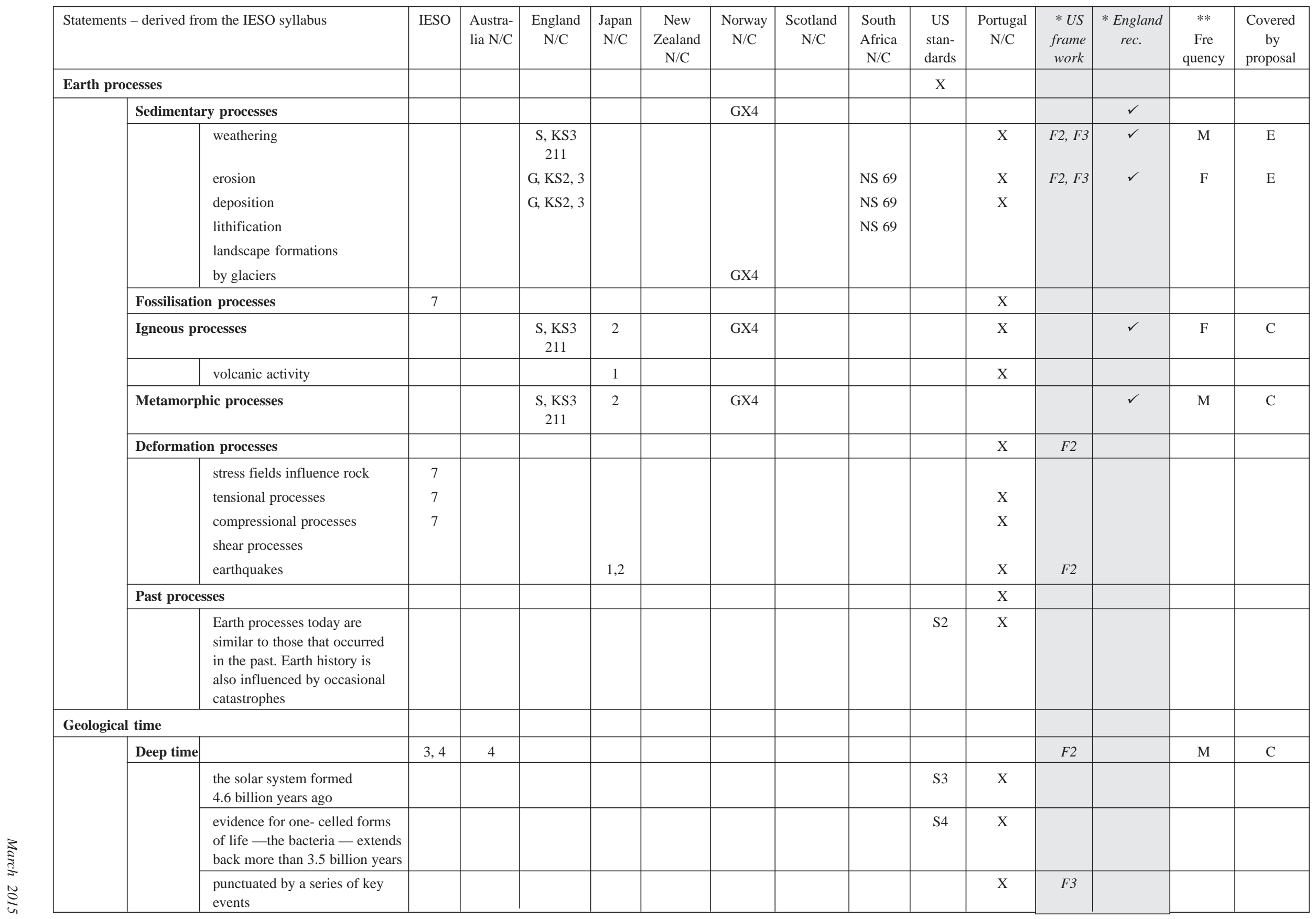


Table 4. Contd....

\begin{tabular}{|c|c|c|c|c|c|c|c|c|c|c|c|c|c|c|}
\hline Statements - derived from the IESO syllabus & IESO & $\begin{array}{l}\text { Austra- } \\
\text { lia N/C }\end{array}$ & $\begin{array}{l}\text { England } \\
\mathrm{N} / \mathrm{C}\end{array}$ & $\begin{array}{c}\text { Japan } \\
\text { N/C }\end{array}$ & $\begin{array}{l}\text { New } \\
\text { Zealand } \\
\text { N/C }\end{array}$ & $\begin{array}{l}\text { Norway } \\
\text { N/C }\end{array}$ & $\begin{array}{l}\text { Scotland } \\
\text { N/C }\end{array}$ & $\begin{array}{l}\text { South } \\
\text { Africa } \\
\text { N/C }\end{array}$ & $\begin{array}{l}\text { US } \\
\text { stan- } \\
\text { dards }\end{array}$ & $\begin{array}{c}\text { Portugal } \\
\text { N/C }\end{array}$ & $\begin{array}{l}* \text { US } \\
\text { frame } \\
\text { work }\end{array}$ & $\begin{array}{l}\text { * England } \\
\text { rec. }\end{array}$ & $\begin{array}{c}* * \\
\text { Fre } \\
\text { quency }\end{array}$ & $\begin{array}{l}\text { Covered } \\
\text { by } \\
\text { proposal }\end{array}$ \\
\hline Relative dating & & & & & 2 & & & & & & & & & \\
\hline $\begin{array}{l}\text { using rock strata } \\
\text { using fossils } \\
\text { using tree rings and ice cores }\end{array}$ & & & & & & $\begin{array}{l}\text { GX4 } \\
\text { GX4 }\end{array}$ & & & $\begin{array}{l}\text { S3 } \\
\text { S3 }\end{array}$ & $\begin{array}{l}\mathrm{X} \\
\mathrm{X}\end{array}$ & $\begin{array}{l}F 2 \\
F 2 \\
F 2\end{array}$ & $\checkmark$ & $\begin{array}{l}\mathrm{F} \\
\mathrm{M}\end{array}$ & $\begin{array}{l}\mathrm{E} \\
\mathrm{E}\end{array}$ \\
\hline $\begin{array}{l}\text { Absolute dating } \\
\text { by radioactive decay of isotopes }\end{array}$ & & & & & 2 & GX4 & & & S3 & & $F 3$ & $\checkmark$ & $\mathrm{M}$ & E \\
\hline \multicolumn{15}{|l|}{ Structure of the Earth } \\
\hline Characteristics & & & & & & & & & & & & & & \\
\hline $\begin{array}{l}\text { shape and size of the Earth } \\
\text { Earth magnetism }\end{array}$ & & & & $\begin{array}{l}1 \\
1\end{array}$ & & & & & & $\begin{array}{l}\mathrm{X} \\
\mathrm{X}\end{array}$ & & & & \\
\hline Layers & 6 & & & 1 & & & & & S1 & $\mathrm{X}$ & & & M & $\mathrm{C}$ \\
\hline $\begin{array}{l}\text { crust } \\
\text { mantle } \\
\text { core } \\
\text { lithosphere } \\
\text { evidence for Earth's structure } \\
\text { (probes, seismic, magnetic, } \\
\text { geological) }\end{array}$ & 6 & & & & & & & $\begin{array}{l}\text { NS } 71 \\
\text { NS } 71 \\
\text { NS } 71\end{array}$ & S1 & $\begin{array}{l}X \\
X \\
X \\
X \\
X\end{array}$ & $\begin{array}{l}F 3 \\
F 3 \\
F 3 \\
F 3\end{array}$ & $\begin{array}{l}\checkmark \\
\checkmark \\
\checkmark\end{array}$ & $\begin{array}{l}\mathrm{M} \\
\mathrm{M} \\
\mathrm{M}\end{array}$ & $\begin{array}{l}\mathrm{C} \\
\mathrm{C} \\
\mathrm{C}\end{array}$ \\
\hline \multicolumn{15}{|l|}{ Earth cycles } \\
\hline Cyclic processes & & & & & & & & & & & & & & \\
\hline $\begin{array}{l}\text { transition between reservoirs } \\
\text { where form changes but total } \\
\text { amount of matter remains } \\
\text { constant } \\
\text { feedback (positive and negative) } \\
\text { tight coupling of systems } \\
\text { rates vary from fast to very slow }\end{array}$ & $\begin{array}{l}6 \\
6 \\
6 \\
6 \\
6 \\
\end{array}$ & 3 & & & & & & NS 69 & $\mathrm{~S} 1$ & & $\begin{array}{l}F 3 \\
F 2 \\
\end{array}$ & $\checkmark$ & $\begin{array}{l}\mathrm{M} \\
\mathrm{F}\end{array}$ & $\begin{array}{l}\mathrm{C} \\
\mathrm{E}\end{array}$ \\
\hline Earth systems & 6 & 4 & & & 2 & & & & S3 & & $F 3$ & $\checkmark$ & $\mathrm{F}$ & $\mathrm{C}$ \\
\hline $\begin{array}{l}\text { lithosphere } \\
\text { hydrosphere } \\
\text { atmosphere } \\
\text { biosphere }\end{array}$ & $\begin{array}{l}6 \\
6 \\
6 \\
6\end{array}$ & $\begin{array}{l}4 \\
4 \\
4 \\
4\end{array}$ & & & & & & $\begin{array}{l}\text { NS } 71 \\
\text { NS } 71 \\
\text { NS } 71\end{array}$ & & & $\begin{array}{l}F 3 \\
F 3 \\
F 3 \\
F 3\end{array}$ & $\begin{array}{l}\checkmark \\
\checkmark \\
\checkmark \\
\checkmark\end{array}$ & $\begin{array}{l}\mathrm{F} \\
\mathrm{F} \\
\mathrm{F} \\
\mathrm{M}\end{array}$ & $\begin{array}{l}\mathrm{C} \\
\mathrm{C} \\
\mathrm{C} \\
\mathrm{C}\end{array}$ \\
\hline
\end{tabular}


Table 4. Contd....

\begin{tabular}{|c|c|c|c|c|c|c|c|c|c|c|c|c|c|c|}
\hline Statements - derived from the IESO syllabus & IESO & $\begin{array}{l}\text { Austra- } \\
\text { lia N/C }\end{array}$ & $\begin{array}{c}\text { England } \\
\text { N/C }\end{array}$ & $\begin{array}{c}\text { Japan } \\
\mathrm{N} / \mathrm{C}\end{array}$ & $\begin{array}{c}\text { New } \\
\text { Zealand } \\
\text { N/C }\end{array}$ & $\begin{array}{l}\text { Norway } \\
\text { N/C }\end{array}$ & $\begin{array}{l}\text { Scotland } \\
\text { N/C }\end{array}$ & $\begin{array}{c}\text { South } \\
\text { Africa } \\
\text { N/C }\end{array}$ & $\begin{array}{l}\text { US } \\
\text { stan- } \\
\text { dards }\end{array}$ & $\begin{array}{l}\text { Portugal } \\
\text { N/C }\end{array}$ & $\begin{array}{l}* \text { US } \\
\text { frame } \\
\text { work }\end{array}$ & $\begin{array}{l}\text { *England } \\
\text { rec. }\end{array}$ & $\begin{array}{c}* * \\
\text { Fre } \\
\text { quency }\end{array}$ & $\begin{array}{c}\text { Covered } \\
\text { by } \\
\text { proposal }\end{array}$ \\
\hline \multicolumn{15}{|l|}{ Earth cycles } \\
\hline Earth systems & & & & & & & & & & & & & & \\
\hline $\begin{array}{l}\text { interactions and cycles within } \\
\text { and between Earth's spheres }\end{array}$ & & 4 & & & & & & & & & & $\checkmark$ & & \\
\hline $\begin{array}{l}\text { the many dynamic and delicate } \\
\text { feedbacks between the biosphere } \\
\text { and other Earth systems cause } \\
\text { a continual co-evolution of } \\
\text { Earth's surface and the life } \\
\text { that exists on it }\end{array}$ & & & & & & & & & S3 & & F6 & $\checkmark$ & M & $\mathrm{E}$ \\
\hline Earth energy sources & 6 & & & & & & & & & & & & & \\
\hline solar energy & 6 & & & & & & & NS 71 & S3 & $\mathrm{X}$ & $F 3$ & & $\mathrm{~F}$ & $\mathrm{E}$ \\
\hline $\begin{array}{l}\text { internal energy (including } \\
\text { radioactive decay) }\end{array}$ & 6 & & & & & & & & S3 & & $F 3$ & & & \\
\hline $\begin{array}{l}\text { gravitational energy from the } \\
\text { Earth's original formation. }\end{array}$ & & & & & & & & & S3 & & & & & \\
\hline Rock cycle & 6 & & $\begin{array}{l}\text { S, KS3 } \\
211\end{array}$ & & & & & & S2 & $X$ & & & M & C \\
\hline Plate tectonic cycle & 6 & 4 & $\begin{array}{l}\text { S, KS4 } \\
225\end{array}$ & 2 & & & & & & $\mathrm{X}$ & & & F & $\mathrm{C}$ \\
\hline $\begin{array}{l}\text { lithospheric plates constantly } \\
\text { move at rates of centimetres } \\
\text { per year in response to move- } \\
\text { ments in the mantle. Major } \\
\text { geological events, such as } \\
\text { earthquakes, volcanic eruptions, } \\
\text { and mountain building, result } \\
\text { from these plate motions. }\end{array}$ & & & & 1 & & & & NS 71 & S1 & $\mathrm{x}$ & & $\checkmark$ & F & $\mathrm{C}$ \\
\hline $\begin{array}{l}\text { tectonic processes continually } \\
\text { generate new ocean seafloor at } \\
\text { ridges and destroy old seafloor } \\
\text { at trenches }\end{array}$ & & & & & & & & & & & $F 3$ & $\checkmark$ & & \\
\hline
\end{tabular}


Table 4. Contd....

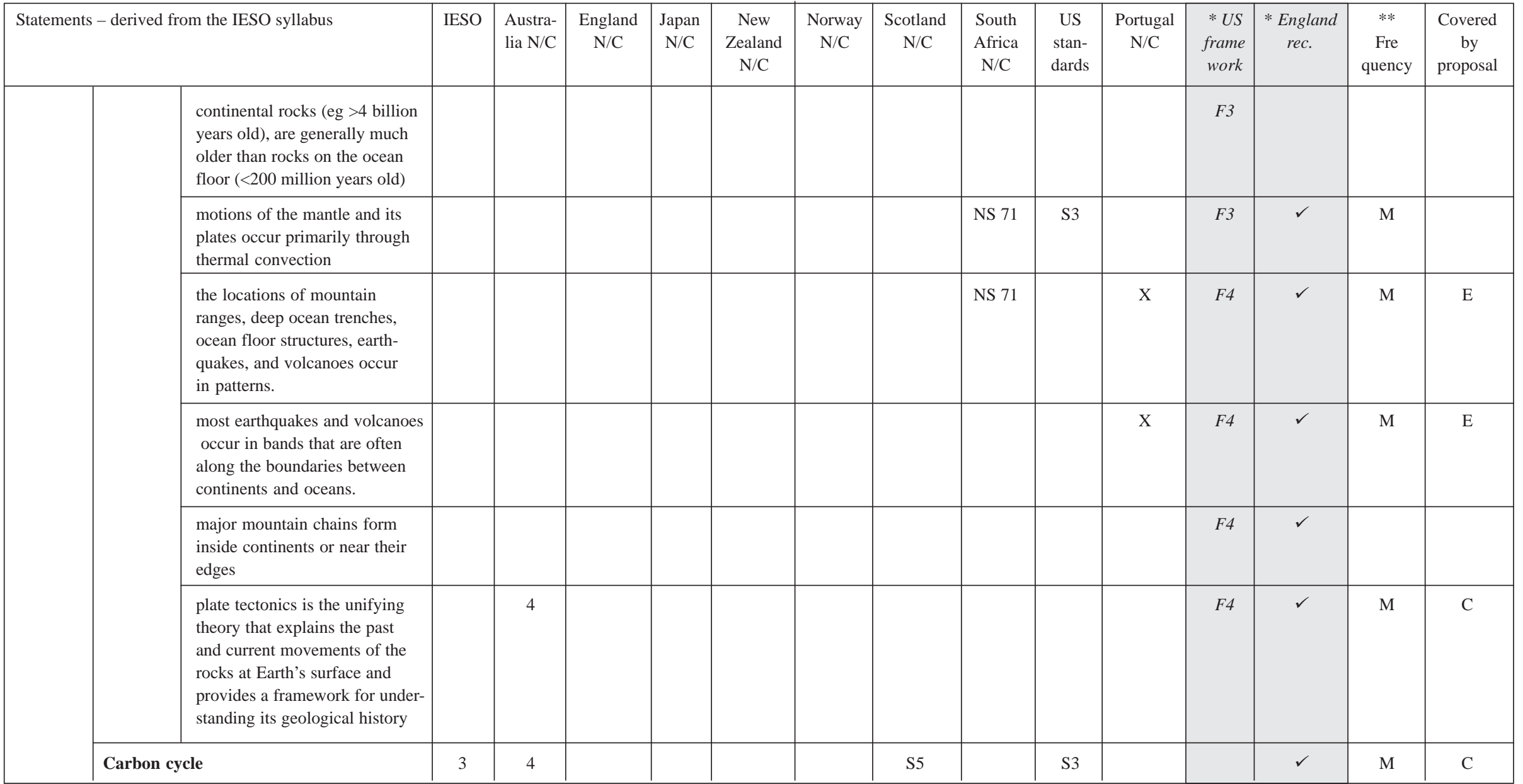


Table 5. Matrix of coverage by current school-level Earth science syllabuses - the hydrosphere

\begin{tabular}{|c|c|c|c|c|c|c|c|c|c|c|c|c|c|c|}
\hline Statements - derived from the IESO syllabus & IESO & $\begin{array}{l}\text { Austra- } \\
\text { lia N/C }\end{array}$ & $\begin{array}{l}\text { England } \\
\text { N/C }\end{array}$ & $\begin{array}{c}\text { Japan } \\
\text { N/C }\end{array}$ & $\begin{array}{l}\text { New } \\
\text { Zealand } \\
\text { N/C }\end{array}$ & $\begin{array}{l}\text { Norway } \\
\text { N/C }\end{array}$ & $\begin{array}{l}\text { Scotland } \\
\text { N/C }\end{array}$ & $\begin{array}{l}\text { South } \\
\text { Africa } \\
\text { N/C }\end{array}$ & $\begin{array}{l}\text { US } \\
\text { stan- } \\
\text { dards }\end{array}$ & $\begin{array}{l}\text { Portugal } \\
\text { N/C }\end{array}$ & $\begin{array}{l}* U S \\
\text { frame } \\
\text { work }\end{array}$ & $\begin{array}{l}* \text { England } \\
\text { rec. }\end{array}$ & $\begin{array}{l}* * \\
\text { Fre } \\
\text { quency }\end{array}$ & $\begin{array}{l}\text { Covered } \\
\text { by } \\
\text { proposal }\end{array}$ \\
\hline The hydrosphere & 8 & 4 & & & & & & & & & & & & \\
\hline Water & 8 & & & & & & & & & & & & & \\
\hline $\begin{array}{l}\text { water cycle (transpiration, } \\
\text { evaporation, condensation and } \\
\text { crystallization, and precipitation } \\
\text { as well as downhill flows on land) }\end{array}$ & & 3 & & & 3 & & S5 & NS 70 & $\mathrm{~S} 2$ & & F5 & $\checkmark$ & $\mathrm{F}$ & $\mathrm{C}$ \\
\hline $\begin{array}{l}\text { the geosphere, hydrosphere, } \\
\text { atmosphere and biosphere are } \\
\text { tightly coupled }\end{array}$ & 8 & & & & & & & & & & $F 3$ & & & \\
\hline $\begin{array}{l}\text { natural water composition is } \\
\text { affected by geological processes }\end{array}$ & 8 & & & & & & & & & & & & & \\
\hline $\begin{array}{l}\text { water's unique combination of } \\
\text { physical and chemical properties } \\
\text { are central to the planet's dynamics. } \\
\text { These properties include water's } \\
\text { exceptional capacity to absorb, } \\
\text { store, and release large amounts of } \\
\text { energy; transmit sunlight; expand } \\
\text { upon freezing; dissolve and transport } \\
\text { materials; and lower the viscosities } \\
\text { and melting points of rocks }\end{array}$ & 8 & & & & 4 & & & & & & $F 5$ & & M & $\mathrm{E}$ \\
\hline $\begin{array}{l}\text { water availability is affected by } \\
\text { atmospheric and geological processes }\end{array}$ & 8 & & & & & & & & & & F5 & & & \\
\hline $\begin{array}{l}\text { the amount of water for human } \\
\text { consumption is limited }\end{array}$ & 8 & & & & & & & & & & & & & \\
\hline $\begin{array}{l}\text { water resources need to be carefully } \\
\text { managed }\end{array}$ & & & & & & & & NS 70 & & & & & & \\
\hline flood & 8 & & & & & GX4 & & & & & & $\checkmark$ & $\mathrm{M}$ & \\
\hline drought & & & G, KS2, 3 & & & GX4 & & & & & & & & \\
\hline Oceanic water & 8 & & & & & & & & & & & & & \\
\hline $\begin{array}{l}\text { oceanic water composition affected } \\
\text { by geological processes }\end{array}$ & 8 & & & & 4 & & & & & & & & & \\
\hline $\begin{array}{l}\text { the composition of oceanic water } \\
\text { evolved over geological time } \\
\text { ocean currents are the result of }\end{array}$ & 8 & & & & & & & & & & & $\checkmark$ & & \\
\hline $\begin{array}{l}\text { unequal heating of the Earth and } \\
\text { salinity differences }\end{array}$ & 9 & & & 1 & & & & & & & F5 & $\checkmark$ & M & $\mathrm{C}$ \\
\hline $\begin{array}{l}\text { the ocean exerts a major influence } \\
\text { on weather and climate by absorbing } \\
\text { energy from the sun, releasing it } \\
\text { over time, and globally redistributing } \\
\text { it through ocean currents. }\end{array}$ & & & & 2 & 2,4 & & & & & & $F 5$ & & M & $\mathrm{E}$ \\
\hline tsunamis & 8 & & & & & & & & & & & & & \\
\hline tides & & & & & & & & & & & & & & \\
\hline storms (hurricance and typhoons) & 8 & & & & & & & & & & & & & \\
\hline
\end{tabular}


Table 6. Matrix of coverage by current school-level Earth science syllabuses - the atmosphere

\begin{tabular}{|c|c|c|c|c|c|c|c|c|c|c|c|c|c|c|c|}
\hline \multicolumn{2}{|c|}{ Statements - derived from the IESO syllabus } & IESO & $\begin{array}{l}\text { Austra- } \\
\text { lia N/C }\end{array}$ & $\begin{array}{l}\text { England } \\
\text { N/C }\end{array}$ & $\begin{array}{c}\text { Japan } \\
\text { N/C }\end{array}$ & $\begin{array}{l}\text { New } \\
\text { Zealand } \\
\text { N/C }\end{array}$ & $\begin{array}{l}\text { Norway } \\
\text { N/C }\end{array}$ & $\begin{array}{c}\text { Scotland } \\
\text { N/C }\end{array}$ & $\begin{array}{l}\text { South } \\
\text { Africa } \\
\text { N/C }\end{array}$ & $\begin{array}{l}\text { US } \\
\text { stan- } \\
\text { dards }\end{array}$ & $\begin{array}{l}\text { Portugal } \\
\text { N/C }\end{array}$ & $\begin{array}{l}\text { *US } \\
\text { frame } \\
\text { work }\end{array}$ & $\begin{array}{l}* \text { England } \\
\text { rec. }\end{array}$ & $\begin{array}{c}* * \\
\text { Fre } \\
\text { quency }\end{array}$ & $\begin{array}{c}\text { Covered } \\
\text { by } \\
\text { proposal }\end{array}$ \\
\hline \multicolumn{2}{|l|}{ The atmosphere } & 8 & 4 & & & & & & & & & & & & \\
\hline \multicolumn{2}{|c|}{ Composition } & 9 & & & & & & & & & & & & & \\
\hline & $\begin{array}{l}\text { the geosphere, hydrosphere, } \\
\text { atmosphere and biosphere are } \\
\text { tightly coupled }\end{array}$ & 9 & & & & & & & & & & & & & \\
\hline & $\begin{array}{l}\text { the composition of the early } \\
\text { atmosphere was from gases omitted } \\
\text { by volcanic activity }\end{array}$ & 9 & & & & & & & & & & & & & \\
\hline & $\begin{array}{l}\text { the composition of the atmosphere } \\
\text { has evolved over geological time }\end{array}$ & 9 & & $\begin{array}{l}\text { S, KS4 } \\
225\end{array}$ & & & & & & & & & $\checkmark$ & M & $\mathrm{C}$ \\
\hline & $\begin{array}{l}\text { evolution of the composition of the } \\
\text { atmosphere is tightly linked to } \\
\text { evolution of life on Earth }\end{array}$ & 9 & & & & & & & & $\mathrm{~S} 4$ & & & $\checkmark$ & & \\
\hline & $\begin{array}{l}\text { the modern atmosphere is a mixture } \\
\text { of nitrogen, oxygen, and trace gases } \\
\text { that include water vapour }\end{array}$ & & & & & 4 & & S5 & $\begin{array}{l}\text { NS } \\
71\end{array}$ & S2 & & & $\checkmark$ & $\mathrm{F}$ & $\mathrm{C}$ \\
\hline & $\begin{array}{l}\text { the atmosphere protects the earth } \\
\text { from harmful radiation and from most } \\
\text { objects from outer space that would } \\
\text { otherwise strike the Earth's surface }\end{array}$ & & & & & & & & $\begin{array}{r}\text { NS } \\
71\end{array}$ & & & & & & \\
\hline \multicolumn{2}{|l|}{ Flow } & 9 & & & & & & & & & & & & & \\
\hline & $\begin{array}{l}\text { the foundation for Earth's global } \\
\text { climate system is the electromagnetic } \\
\text { radiation from the sun as well as its } \\
\text { reflection, absorption, storage, and } \\
\text { redistribution among the atmosphere, } \\
\text { ocean, and land systems and this } \\
\text { energy's re-radiation into space. }\end{array}$ & & & & 1 & 2 & SS8 & & & & & $F 6$ & & M & $\mathrm{C}$ \\
\hline & $\begin{array}{l}\text { Earth's axial tilt causes differential } \\
\text { intensity of sunlight on different } \\
\text { areas of Earth }\end{array}$ & & & & & & & & & S3 & & $F 2$ & & & \\
\hline & $\begin{array}{l}\text { atmospheric flows are the result of } \\
\text { unequal heating of the Earth }\end{array}$ & 9 & & & 1,2 & & & & & S3 & & & $\checkmark$ & $\mathrm{M}$ & $\mathrm{E}$ \\
\hline \multirow[t]{3}{*}{ Change } & & & & & & & & & & & & & & & \\
\hline & climate change & 1,2 & & $\begin{array}{c}\text { G, KS3 } \\
106\end{array}$ & & & & S5 & & & & & $\checkmark$ & M & C \\
\hline & $\begin{array}{l}\text { global climate is determined by } \\
\text { energy transfer from the sun at and } \\
\text { near the earth's surface. This energy } \\
\text { transfer is influenced by dynamic } \\
\text { processes such as cloud cover and the } \\
\text { Earth's rotation, and static conditions } \\
\text { such as the position of mountain } \\
\text { ranges and oceans }\end{array}$ & S3 & & & & & GX4 & & & & & & & & \\
\hline
\end{tabular}


Table 6. Contd....

\begin{tabular}{|c|c|c|c|c|c|c|c|c|c|c|c|c|c|c|c|}
\hline \multicolumn{2}{|c|}{ Statements - derived from the IESO syllabus } & \multirow[t]{2}{*}{ IESO } & \multirow{2}{*}{$\begin{array}{l}\text { Austra- } \\
\text { lia N/C }\end{array}$} & \multirow{2}{*}{$\begin{array}{l}\text { England } \\
\text { N/C }\end{array}$} & \multirow{2}{*}{$\begin{array}{c}\text { Japan } \\
\text { N/C }\end{array}$} & \multirow{2}{*}{$\begin{array}{c}\text { New } \\
\text { Zealand } \\
\text { N/C } \\
2\end{array}$} & \multirow{2}{*}{$\begin{array}{c}\text { Norway } \\
\text { N/C }\end{array}$} & \multirow{2}{*}{$\begin{array}{l}\text { Scotland } \\
\text { N/C }\end{array}$} & \multirow{2}{*}{$\begin{array}{c}\text { South } \\
\text { Africa } \\
\text { N/C }\end{array}$} & \multirow{2}{*}{$\begin{array}{l}\text { US } \\
\text { stan- } \\
\text { dards }\end{array}$} & \multirow{2}{*}{$\begin{array}{c}\text { Portugal } \\
\text { N/C }\end{array}$} & \multirow{2}{*}{$\begin{array}{r}\text { *US } \\
\text { frame } \\
\text { work } \\
F 2\end{array}$} & \multirow{2}{*}{$\begin{array}{l}\text { * England } \\
\text { rec. }\end{array}$} & \multirow{2}{*}{$\begin{array}{c}* * \\
\text { Fre } \\
\text { quency }\end{array}$} & \multirow{2}{*}{$\begin{array}{c}\text { Covered } \\
\text { by } \\
\text { proposal }\end{array}$} \\
\hline \multirow[t]{6}{*}{ Change } & $\begin{array}{l}\text { cyclical changes in the shape of } \\
\text { Earth's orbit around the sun, } \\
\text { together with changes in the } \\
\text { orientation of the planet's axis of } \\
\text { rotation, both occurring over tens } \\
\text { to hundreds of thousands of years, } \\
\text { have altered the intensity and } \\
\text { distribution of sunlight falling on } \\
\text { Earth. These phenomena cause } \\
\text { cycles of ice ages and other gradual } \\
\text { climate changes }\end{array}$ & & & & & & & & & & & & & & \\
\hline & $\begin{array}{l}\text { greenhouse gases in the atmosphere } \\
\text { absorb and retain the energy radiated } \\
\text { from land and ocean surfaces, } \\
\text { thereby regulating Earth's average } \\
\text { surface temperature and keeping it } \\
\text { habitable. }\end{array}$ & & & & & & S8 & & & & & F5 & $\checkmark$ & M & $\mathrm{C}$ \\
\hline & $\begin{array}{l}\text { climate change can occur when } \\
\text { certain parts of Earth's systems are } \\
\text { altered. Geological evidence indicates } \\
\text { that past climate changes were } \\
\text { either sudden changes caused by } \\
\text { alterations in the atmosphere; } \\
\text { longer term changes (e.g., ice ages) } \\
\text { due to variations in solar output, } \\
\text { Earth's orbit, or the orientation of } \\
\text { its axis; or even more gradual } \\
\text { atmospheric changes due to plants } \\
\text { and other organisms that captured } \\
\text { carbon dioxide and released oxygen. } \\
\text { The time scales of these changes } \\
\text { varied from a few to millions of } \\
\text { years }\end{array}$ & & & & & & & & & & & F6 & & & \\
\hline & $\begin{array}{l}\text { changes in the atmosphere due to } \\
\text { human activity have increased } \\
\text { carbon dioxide concentrations } \\
\text { and thus affect climate }\end{array}$ & & & & & & & & & & & F6 & & & \\
\hline & $\begin{array}{l}\text { if Earth's global mean temperature } \\
\text { continues to rise, the lives of humans } \\
\text { and other organisms will be affected } \\
\text { in many different ways }\end{array}$ & & & & & & & S5 & & & & $F 8$ & & & \\
\hline & $\begin{array}{l}\text { global climate models are often used } \\
\text { to understand the process of climate } \\
\text { change }\end{array}$ & & & & & & & & & & & $F 8$ & & & \\
\hline
\end{tabular}


Table 7. Matrix of coverage by current school-level Earth science syllabuses - the biosphere

\begin{tabular}{|c|c|c|c|c|c|c|c|c|c|c|c|c|c|c|}
\hline Statements - derived from the IESO syllabus & IESO & $\begin{array}{l}\text { Austra- } \\
\text { lia N/C }\end{array}$ & $\begin{array}{l}\text { England } \\
\text { N/C }\end{array}$ & $\begin{array}{c}\text { Japan } \\
\text { N/C }\end{array}$ & $\begin{array}{c}\text { New } \\
\text { Zealand } \\
\text { N/C }\end{array}$ & $\begin{array}{c}\text { Norway } \\
\text { N/C }\end{array}$ & $\begin{array}{c}\text { Scotland } \\
\text { N/C }\end{array}$ & $\begin{array}{c}\text { South } \\
\text { Africa } \\
\text { N/C }\end{array}$ & $\begin{array}{c}\text { US } \\
\text { stan- } \\
\text { dards }\end{array}$ & $\begin{array}{l}\text { Portugal } \\
\text { N/C }\end{array}$ & $\begin{array}{l}* U S \\
\text { frame } \\
\text { work }\end{array}$ & $\begin{array}{l}\text { * England } \\
\text { rec. }\end{array}$ & $\begin{array}{l}* * \\
\text { Fre } \\
\text { quency }\end{array}$ & $\begin{array}{c}\text { Covered } \\
\text { by } \\
\text { proposal }\end{array}$ \\
\hline The biosphere & & 4 & & & & & & & & & & & & \\
\hline \multicolumn{15}{|l|}{ Interactions } \\
\hline $\begin{array}{l}\text { the evolution and proliferation of living } \\
\text { things over geological time have in turn } \\
\text { changed the rates of weathering and erosion } \\
\text { of land surfaces, altered the composition of } \\
\text { Earth's soils and atmosphere, and affected } \\
\text { the distribution of water in the hydrosphere. }\end{array}$ & 3,6 & & & & & & & & & & F6 & & & \\
\hline Evolution & & & 5 & & & & & & & & & & & \\
\hline fossils provide evidence for evolution & & & & & & & & NS 63 & & & & $\checkmark$ & & \\
\hline $\begin{array}{l}\text { evolution is shaped by Earth's varying } \\
\text { geological conditions }\end{array}$ & & & & & & & & & & & $F 7$ & $\checkmark$ & & \\
\hline $\begin{array}{l}\text { sudden changes in conditions (e.g., meteor } \\
\text { impacts, major volcanic eruptions) have } \\
\text { caused mass extinctions, but these changes, } \\
\text { as well as more gradual ones, have ultimately } \\
\text { allowed other life forms to flourish }\end{array}$ & & & & & & & & & & & F7 & & & \\
\hline $\begin{array}{l}\text { evidence for theories that explain the } \\
\text { diversity of life on Earth and evolution }\end{array}$ & & 5 & & & & & & & & & & $\checkmark$ & & \\
\hline
\end{tabular}

Table 8. Matrix of coverage by current school-level Earth science syllabuses - the solar system

\begin{tabular}{|c|c|c|c|c|c|c|c|c|c|c|c|c|c|c|}
\hline Statements - derived from the IESO syllabus & IESO & $\begin{array}{l}\text { Austra- } \\
\text { lia N/C }\end{array}$ & $\begin{array}{l}\text { England } \\
\mathrm{N} / \mathrm{C}\end{array}$ & $\begin{array}{c}\text { Japan } \\
\text { N/C }\end{array}$ & $\begin{array}{l}\text { New } \\
\text { Zealand } \\
\mathrm{N} / \mathrm{C}\end{array}$ & $\begin{array}{l}\text { Norway } \\
\text { N/C }\end{array}$ & $\begin{array}{l}\text { Scotland } \\
\text { N/C }\end{array}$ & $\begin{array}{l}\text { South } \\
\text { Africa } \\
\text { N/C }\end{array}$ & $\begin{array}{l}\text { US } \\
\text { stan- } \\
\text { dards }\end{array}$ & $\begin{array}{l}\text { Portugal } \\
\text { N/C }\end{array}$ & $\begin{array}{l}* \text { US } \\
\text { frame } \\
\text { work }\end{array}$ & $\begin{array}{l}* \text { England } \\
\text { rec. }\end{array}$ & $\begin{array}{c}* * \\
\text { Fre } \\
\text { quency }\end{array}$ & $\begin{array}{l}\text { Covered } \\
\text { by } \\
\text { proposal }\end{array}$ \\
\hline The solar system & & & & & & & & & & $\mathrm{X}$ & & & & \\
\hline Planetary system & & 10 & & & & 3 & & & & & & & & \\
\hline $\begin{array}{l}\text { the star called the sun is changing } \\
\text { and will burn out over a life span of } \\
\text { approximately } 10 \text { billion years }\end{array}$ & & & & & & & & & S3 & & $F 1$ & & & \\
\hline $\begin{array}{l}\text { Earth rotation, day/night, daily/ } \\
\text { seasonal changes, phases of moon }\end{array}$ & & 1,3 & & 2 & & S4, S6 & S6 & NS 69 & $\begin{array}{l}\mathrm{S} 1, \mathrm{~S} 2, \\
\quad \mathrm{~S} 3\end{array}$ & & $F 2$ & $\checkmark$ & $\mathrm{F}$ & $\mathrm{C}$ \\
\hline eclipses & & 3 & & & & & & & S2 & & $F 2$ & & $\mathrm{M}$ & $\mathrm{C}$ \\
\hline tides & & & & & & & & & S2 & & $F 2$ & & & \\
\hline $\begin{array}{l}\text { Earth systems are a subset of } \\
\text { planetary systems }\end{array}$ & 10 & & & & & & & & & & & & & \\
\hline $\begin{array}{l}\text { energy balances of planets include } \\
\text { external (solar) energy and internal } \\
\text { energy }\end{array}$ & 10 & & & & & & & & & & & & & \\
\hline the solar system has evolved over & & & S, KS & & & & & & & & & & & \\
\hline time & & & 4225 & & & & & & & & & & & \\
\hline
\end{tabular}


Table 9. Matrix of coverage by current school-level Earth science syllabuses - environmental geoscience

\begin{tabular}{|c|c|c|c|c|c|c|c|c|c|c|c|c|c|c|}
\hline Statements - derived from the IESO syllabus & IESO & $\begin{array}{l}\text { Austra- } \\
\text { lia N/C }\end{array}$ & $\begin{array}{l}\text { England } \\
\text { N/C }\end{array}$ & $\begin{array}{c}\text { Japan } \\
\text { N/C }\end{array}$ & $\begin{array}{l}\text { New } \\
\text { Zealand } \\
\text { N/C }\end{array}$ & $\begin{array}{c}\text { Norway } \\
\mathrm{N} / \mathrm{C}\end{array}$ & $\begin{array}{l}\text { Scotland } \\
\text { N/C }\end{array}$ & $\begin{array}{l}\text { South } \\
\text { Africa } \\
\text { N/C }\end{array}$ & $\begin{array}{l}\text { US } \\
\text { stan- } \\
\text { dards }\end{array}$ & $\begin{array}{l}\text { Portugal } \\
\text { N/C }\end{array}$ & $\begin{array}{l}* U S \\
\text { frame } \\
\text { work }\end{array}$ & $\begin{array}{l}\text { * England } \\
\text { rec. }\end{array}$ & $\begin{array}{c}* * \\
\text { Fre } \\
\text { quency }\end{array}$ & $\begin{array}{c}\text { Covered } \\
\text { by } \\
\text { proposal }\end{array}$ \\
\hline \multicolumn{15}{|l|}{ Environmental geoscience } \\
\hline $\begin{array}{l}\text { global distribution of resources depends } \\
\text { upon past geological processes }\end{array}$ & & & & & & & & & & & & $\checkmark$ & & \\
\hline $\begin{array}{l}\text { the environment is part of a cyclic world } \\
\text { formed of sub-systems (geosphere, hydro- } \\
\text { sphere, atmosphere and biosphere) that } \\
\text { coexist }\end{array}$ & 2 & & & & & & & & & & & & & \\
\hline $\begin{array}{l}\text { humans are an integral part of the natural } \\
\text { system }\end{array}$ & 2 & & $\begin{array}{r}\text { S, KS3 } \\
211 \& \mathrm{G} \\
\mathrm{KS} 3103 \\
\end{array}$ & & 4 & & & & & & $F 3$ & & $\mathrm{M}$ & $\mathrm{C}$ \\
\hline $\begin{array}{l}\text { all materials, energy, and fuels that humans } \\
\text { use are derived from natural sources, and } \\
\text { their use affects the environment in multiple } \\
\text { ways. }\end{array}$ & & & & & & & & NS 72 & & $\mathrm{X}$ & $F 7$ & & M & $\mathrm{C}$ \\
\hline $\begin{array}{l}\text { the effects of human activity on the } \\
\text { environment }\end{array}$ & & & $\begin{array}{l}\text { S, KS4 } \\
225\end{array}$ & & & & & SS 88 & & $\mathrm{X}$ & & & & \\
\hline $\begin{array}{l}\text { explain how crude oil and natural gas have } \\
\text { come about and how these substances } \\
\text { are used }\end{array}$ & & & & & & S8 & S4 & & & & & & & \\
\hline $\begin{array}{l}\text { all forms of energy production and other } \\
\text { resource extraction have associated economic, } \\
\text { social, environmental, and geopolitical } \\
\text { costs and risks, as well as benefits }\end{array}$ & & & & & & SS9 & S4 & SS $92 / 3$ & & $\mathrm{X}$ & $F 7$ & & $\mathrm{~F}$ & $\mathrm{C}$ \\
\hline sustainable development & & 3 & $\begin{array}{c}\text { S, KS3 } \\
212 \& \mathrm{G} \\
\mathrm{KS} 3103\end{array}$ & & & S8 & S4, G6 & $\begin{array}{l}\text { NS } 58 / 9 \text {, } \\
\text { SS } 92\end{array}$ & & $\mathrm{X}$ & F8 & $\checkmark$ & $\mathrm{F}$ & $\mathrm{C}$ \\
\hline renewable and non-renewable resources & & 3 & $\begin{array}{l}\text { S, KS3, } \\
212\end{array}$ & & & S8 & S4 & $\begin{array}{l}\text { NS } 67 \\
72\end{array}$ & & & F6 & $\checkmark$ & $\mathrm{F}$ & $\mathrm{C}$ \\
\hline $\begin{array}{l}\text { environmental problem identification and } \\
\text { suggestion of solutions }\end{array}$ & 4 & 2 & & & & & & G6 & & & $\mathrm{X}$ & F8 & & F C \\
\hline $\begin{array}{l}\text { the cause of natural disasters, including } \\
\text { earthquakes, tsunamis and volcanic } \\
\text { eruptions }\end{array}$ & & 2 & & & 2,4 & GX4 & & G6 & SS 89 & & $\mathrm{X}$ & & $\checkmark$ & F C \\
\hline natural hazard forecasting and mitigation & 5 & & & & & GX4 & & & & & & $F 7$ & & MC \\
\hline
\end{tabular}


Table 10. Sources of information for the matrix of coverage by current school-level Earth science syllabuses given in Tables 3 - 9.

\begin{tabular}{|c|c|c|}
\hline Curriculum & Submission by: & Source details \\
\hline $\begin{array}{l}\text { International } \\
\text { EarthScience } \\
\text { Olympiad }\end{array}$ & $\begin{array}{l}\text { Syllabus submitted by } \\
\text { Nir Orion, Israel }\end{array}$ & $\begin{array}{l}\text { International Earth Science Olympiad (IESO) syllabus, } \\
\text { IESO website: http://ieso2012.gl.fcen.uba.ar/index.php/ieso-downloads/syllabus/ }\end{array}$ \\
\hline Australia & $\begin{array}{l}\text { One document précised and } \\
\text { submitted by Bronte Nichols, } \\
\text { others submitted by Ian Clark } \\
\text { and Greg McNamara }\end{array}$ & $\begin{array}{l}\text { 'Australian Curriculum Science: Summary of Earth and Space Science strand; } \\
\text { prepared by Bronte Nicholls' Australian Curriculum, F - } 10 \text { Curriculum: Science. } \\
\text { Found at: http://www.australiancurriculum.edu.au/Science/Curriculum/F-10 }\end{array}$ \\
\hline England & Chris King & $\begin{array}{l}\text { Geography at KS2 (7-11 year olds): } \\
\text { https://www.gov.uk/government/uploads/system/uploads/attachment_data/file/239044/ } \\
\text { PRIMARY_national_curriculum_-_Geography.pdf } \\
\text { Geography at KS3 (11-14 year olds): } \\
\text { https://www.gov.uk/government/uploads/system/uploads/attachment_data/file/239087/ } \\
\text { SECONDARY_national_curriculum_-_Geography.pdf } \\
\text { Science at KS1 and } 2 \text { (5-11 year olds): } \\
\text { https://www.gov.uk/government/uploads/system/uploads/attachment_data/file/239132/ } \\
\text { PRIMARY_national_curriculum_-_Science.pdf } \\
\text { Science at KS3 (11-14 year olds): https://www.gov.uk/government/uploads/system/uploads/ } \\
\text { attachment_data/file/335174/SECONDARY_national_curriculum_-_Science_220714.pdf } \\
\text { Science at KS4 (14-16 year olds): https://www.gov.uk/government/uploads/system/uploads/ } \\
\text { attachment_data/file/319108 National_Curriculum_Reform_England__Key_Stage_4_ } \\
\text { Science_consultation_document.pdf\%204.pdf } \\
\text { King, C. \& Bilham, N. (2012) Towards a balanced Earth science Curriculum for England - } \\
\text { science and geography perspectives. Teaching Earth Sciences 37.1, 45-48. }\end{array}$ \\
\hline Japan & $\begin{array}{l}\text { List of national curriculum } \\
\text { content submitted by } \\
\text { Ken-ichiro Hisada, Japan }\end{array}$ & 'Geoscience Basics' and 'Geoscience' syllabuses. \\
\hline New Zealand & $\begin{array}{l}\text { Specially prepared document } \\
\text { submitted by Glenn Vallender, } \\
\text { New Zealand }\end{array}$ & $\begin{array}{l}\text { 'Summary of the New Zealand curriculum in science' based on the National Curriculum } \\
\text { documents available on the following internet pages: } \\
\text { http://nzcurriculum.tki.org.nz/Curriculum-documents/The-New-Zealand-Curriculum }\end{array}$ \\
\hline Norway & $\begin{array}{l}\text { Documents submitted by } \\
\text { Merethe Frøyland and } \\
\text { Kari Remmen, Norway }\end{array}$ & $\begin{array}{l}\text { Natural science subject curriculum. Found by searching for "Natural science subject } \\
\text { curriculum" Norway, on a search engine ('S' in Tables 3-9) Social studies subject } \\
\text { curriculum [including geography]. Found by searching for "Social studies subject } \\
\text { curriculum" Norway, on a search engine ('SS' in Tables 3-9) Geosciences - programme } \\
\text { subject in programmes for specialisation in general studies. } \\
\text { From: http://horselshemmet.vilbli.no/?Lan=3\&Program=V.ST\&Side=1.2.1\&Fag= } \\
\text { V.GFG1-01 ( 'GX' in Tables 3-9-GX is studied by all secondary students) }\end{array}$ \\
\hline Scotland & Chris King & $\begin{array}{l}\text { Curriculum for excellence; sciences - experiences and outcomes. } \\
\text { From: http://www.educationscotland.gov.uk/learningteachingandassessment/curriculum } \\
\text { areas/socialstudies/eandos/index.asp (indicated as 'S' above) } \\
\text { Curriculum for excellence; social studies - experiences and outcomes. } \\
\text { From: http://www.educationscotland.gov.uk/learningteachingandassessment/curriculum } \\
\text { areas/sciences/eandos/index.asp (indicated as 'G' above) }\end{array}$ \\
\hline South Africa & $\begin{array}{l}\text { Documents submitted by } \\
\text { Ian McKay, South Africa }\end{array}$ & $\begin{array}{l}\text { Department of Education (2002) Revised national curriculum statement for grades } R-9 \\
\text { (schools): Natural sciences. D of E, Pretoria. ISBN: 1-919917-48-9. ('NS' in Tables 3-9) } \\
\text { Department of Education (2002) Revised national curriculum statement for grades } R-9 \\
\text { (schools): Social sciences. D of E, Pretoria. ISBN: 1-919917-47-0. ('SS' in Tables 3-9) }\end{array}$ \\
\hline $\begin{array}{l}\text { United States } \\
\text { of America }\end{array}$ & $\begin{array}{l}\text { Documents précised and } \\
\text { submitted by Mary Dowse, } \\
\text { USA }\end{array}$ & $\begin{array}{l}\text { National Science Education Standards (1996) National Academy Press: Washington DC } \\
\text { ('S' above) A Framework for K-12 Science Education: Practices, Crosscutting Concepts, } \\
\text { and Core Ideas (2012), National Academies Press http://www.nap.edu/catalog.php? } \\
\text { record_id=13165 ('F' in Tables 3-9) }\end{array}$ \\
\hline Portugal & $\begin{array}{l}\text { Information provided by } \\
\text { Luis Marques and } \\
\text { Clara Vasconcelos }\end{array}$ & Personal communication \\
\hline
\end{tabular}


contrasting points of view and suggesting different approaches and content, colleagues were very constructive, understanding and supportive, with the results shown in Tables 1 and 2.

With this international consensus, it is to be hoped that this curriculum document can be used by curriculum developers, geoscience educators, geoscientists and science and geography educators across the world to develop coherent, well-structured curriculum content in Earth science, flexible enough to be developed in national contexts and therefore with clear relevance to those involved. This then will have the potential to improve teaching and learning in Earth science internationally, developing understanding of the past, present and future of our globe whilst providing crucial background education to the decision-makers of the future.

\section{Acknowledgements}

Key feedback comments were provided by Ian Clark - Australia, Rosely Imbernon - Brazil, Luis Marques - Portugal, Ian McKay -
South Africa, Bronte Nichols - Australia, Glenn Vallender - New Zealand, Clara Vasconcelos - Portugal, Ashvin Wickramasooriya Sri Lanka, Michael Wysession - United States of America; these have had the effect of greatly improving the first draft of the syllabus. Many thanks to all these colleagues for their contributions.

\section{References}

Websites here and in Table 10 last checked in November 2014.

Earth science literacy initiative (2010) Earth science literacy principles. Available at: http://www.earthscienceliteracy.org/

King, C. (2014) International Geoscience Syllabus, to be encountered by all pupils by the age of 16 . Available at: http://www.geoscied. org/about_us.htm

King, C. (2013) Geoscience education across the globe - results of the IUGS-COGE/IGEO survey. Episodes, v. 36, no. 1, pp. 19-30.

King, C. (2012) Third International Geoscience Education Survey: 2012 - with 2013 updates, 2. Available at: http://www.geoscied. org/about_us.htm.

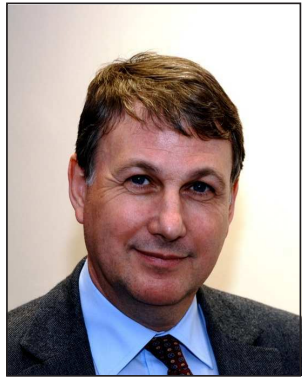

Chris King is Professor of Earth Science Education at Keele University and Director of the Earth Science Education Unit, based at Keele. Following five years as a diamond prospector and an MSc, Chris trained as a teacher and then taught geology and science in a secondary school. Since then, he has been a lecturer at Keele University. He was Chair and is now a Senior Officer/Advisor of the International Geoscience Education Organisation (IGEO). He is Co-Chair of the IUGS Commission on Geoscience Education (IUGS-COGE). He instigated the formation of the Earthlearningidea.com website of international Earth science educational resources and has more than 200 publications. 